\title{
Interference Assessment for the Spectrum Sharing between IMT-2020 and Inter-Satellite Service
}

\author{
Bo $\mathrm{Li}^{1}$, Zhaojun Qian ${ }^{2}$, Shuaijun $\mathrm{Liu}^{1}$,Chaowei Wang ${ }^{1}$, Yinghai Zhang ${ }^{1}$, Weidong Wang ${ }^{1 *}$ \\ ${ }^{1}$ Beijing University of Posts and Telecommunications, Beijing \\ ${ }^{2}$ The State Radio Monitoring Cente, Beijing
}

\section{Abstract}

This paper analyzes interference coexistence of IMT-2020 (International Mobile Telecommunications-2020) and intersatellite service where both systems operate in the same spectrum band of 25.25-27.5GHz. This work can be regarded as a good reference to the research community, industry and regulators which are currently investigating spectrum requirements and technology options for 5G system. Considering the interference scenario between the return inter-orbit link of data relay satellite system and downlink of IMT-2020, we adopt realistic system parameters and radiation pattern, combined with very recent channel from the literature. Simulation results indicate that the interference from IMT-2020 downlink to Data Relay Satellite (DRS) is above the interference level while the LEO spacecraft cause acceptable interference to IMT-2020 downlink.

Keywords: IMT-2020, inter-satellite service, Data Relay Satellite (DRS), spectrum sharing, interference coexistence.

Received on 27 February 2017, accepted on 20 November 2017, published on 10 January 2018

Copyright (C) 2018 Bo Li et al., licensed to EAI. This is an open access article distributed under the terms of the Creative Commons Attribution licence (http://creativecommons.org/licenses/by/3.0/), which permits unlimited use, distribution and reproduction in any medium so long as the original work is properly cited.

doi: 10.4108/eai.10-1-2018.153556

*Corresponding author. Email:wangweidong@bupt.edu.cn

\section{Introduction}

The development of IMT for 2020 and beyond is expected to enable new use cases and rapid traffic growth, for which contiguous and broader channel bandwidths would be desirable. This suggests the need to consider spectrum resources in higher frequency ranges [1]. In order to update frequency allocation decisions and other conditions of use of the radio spectrum at the global level, the World Radiocommunication Conference (WRC) is held every two to four years. WRC-15 agenda revolution 238 considers frequency related matters for the future development of IMT-2020 including possible additional allocations to the inter-satellite services on a primary basis of 25.25-27.5 GHz band. However, this band has been allocated on a co-primary basis to inter-satellite service, thus making it necessary and meaningful to study the coexistence of IMT-2020 and inter-satellite services.
Inter-satellite communication has become an important research topic to improve communication signal system and ranging method [2]. It can ensure high speed transmission and high bandwidths. Moreover, it can be multi-layered network, which supports flexible large scale network structure. The unique advantages of inter-satellite links make it play a more and more important role in military and civil communication field. The 25.25$27.5 \mathrm{GHz}$ band is used by the inter-satellite service for transmission from low-orbiting satellites to receivers onboard geostationary DRS [3]. The sharing between DRS systems and other space and terrestrial radio systems is required in all of the preferred frequency bands, identified in [4]. As the numbers of space and terrestrial radio systems using the same bands will increase in the future, this will increase the potential of interference situations.

25.25-27.5GHz band belongs to the mmWave frequency ranges. Recent studies demonstrate the feasibility of mmWave mobile communications using 
multiple antenna arrays in order to compensate for propagation losses at high frequencies [5]. Study of coexistence between 5G small cells and Fixed Service (FS) at $39 \mathrm{GHz}$ is done in [6], where required frequency rejection is given for tolerable interference on FS resulting from IMT-2020. The spectrum sharing between IMT-2020 and Fixed Satellite Service (FSS) at $28 \mathrm{GHz}$ is simulated in [7]. However, few studies have addressed on spectrum sharing of IMT-2020 and inter-satellite service. It is then important to study the coexistence between inter-satellite service and cellular network to understand whether IMT-2020 operating within these frequencies may affect the inter-satellite service. Similar investigations have been proposed by the International Telecommunication Union (ITU) for spectrum sharing between inter-satellite service and fixed service. The technical and operational requirements that facilitate sharing between point-to-point systems in the fixed service and the inter-satellite service in the 25.2527.5GHz are demonstrated in [8].

Different from the literatures above, we both analyze the interference scenario of terrestrial networks to GSO satellite and LEO spacecraft to terrestrial networks. We consider the propagation model, antenna radiation patterns and parameters provided by ITU, 3GPP and other newest publications. Existing work can be guide on our study, but still many challenges are still undergo. In this work, first we verify the interference scenario of IMT2020 and clarify interference cases in detail. Then we analyze the single point interference and lumped interference. Using the illustrated method, we simulate interference scenario and evaluate interference level. The main contributions of this work is that compared with other $5 \mathrm{G}$ high frequency band, the frequency is relatively low in the band $25.25-27.5 \mathrm{GHz}$ and the device maturity is relatively higher in this band. The research community, industry and community are striving for this band. This work is a research of compatibility analysis between the IMT-2020 system and inter-satellite service.

This paper is organized as follows, Section II describes the model considered focusing on the interference scenario, interference calculation model and propagation model. Section III gives the interference analysis method. Section IV provides the simulation parameters and gives the performance evaluation. Section $\mathrm{V}$ concludes the paper.

\section{System Model}

\subsection{Interference Scenario}

In this section, we discuss reference system for data relay satellite system and sharing scenario between intersatellite service and IMT-2020.

Fig. 1 shows the reference system for data relay satellite system. According to the ITU-R
Recommendation SA.1018 [9], the reference system for data relay system should consist of four cases:

a) The forward feeder link, from the earth station to the data relay satellite.

b) The forward inter orbit link, from the data relay satellite to the low-orbiting spacecraft.

c) The return inter-orbit link, from low-orbiting spacecraft to the data relay satellite.

d) The return feeder link, from the data relay satellite to the earth station.

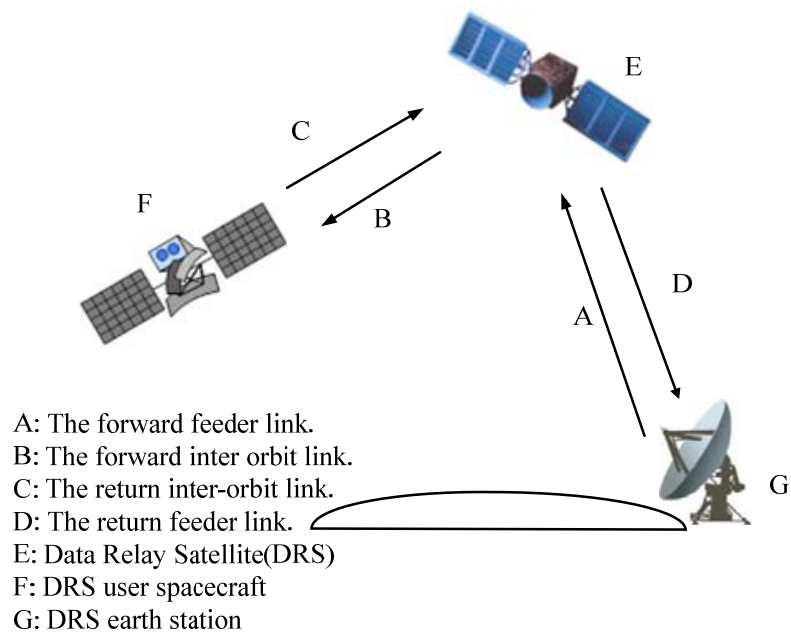

Fig. 1. Data relay satellite system

We assume that IMT-2020 system is sharing the 25.25$27.5 \mathrm{GHz}$ band with inter-satellite service. This frequency range is the operating frequency of the return inter-orbit link where the DRS user spacecraft is in mainly low-earth orbit and the data relay satellite is GEO. To assess whether the frequency band can be allocated to the inter-satellite service and the IMT-2020 system on a primary basis, we consider the interference between IMT-2020 downlink and the return inter-orbit link as is depicted in Fig. 2. Therefore two interference links will be discussed in this paper, that is

e) The interference from IMT-base station to data relay satellite.

f) The interference from DRS spacecraft to the UE.

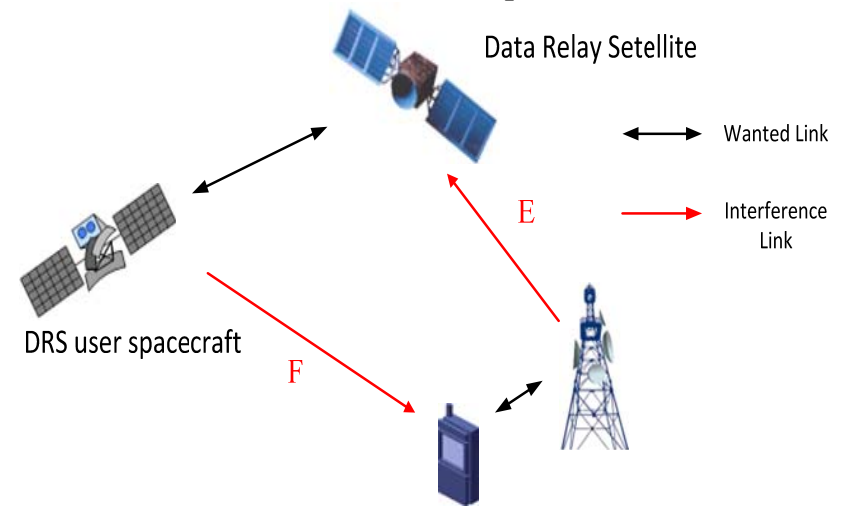

Fig. 2. Sharing scenario between IMT-base station and data relay satellite 


\subsection{IMT-2020 Network Model}

IMT-2020 is a multi-radio access system that will combine LTE and 5G features (i.e., multi-antenna, beamforming, new radio interface, and operation at higher frequencies) [10]. Since the wavelength of millimeter wave frequencies are very small, so it will utilize polarization and different spatial processing techniques like massive MIMO and adaptive beamforming [11]. According to 3GPP, we mainly refer to LTE deployment and the network layout as Fig. 3. The macro cell network is a tri-sector layout placed on a hexagonal grid with distance of $3 R$, where $R$ is the cell radius.

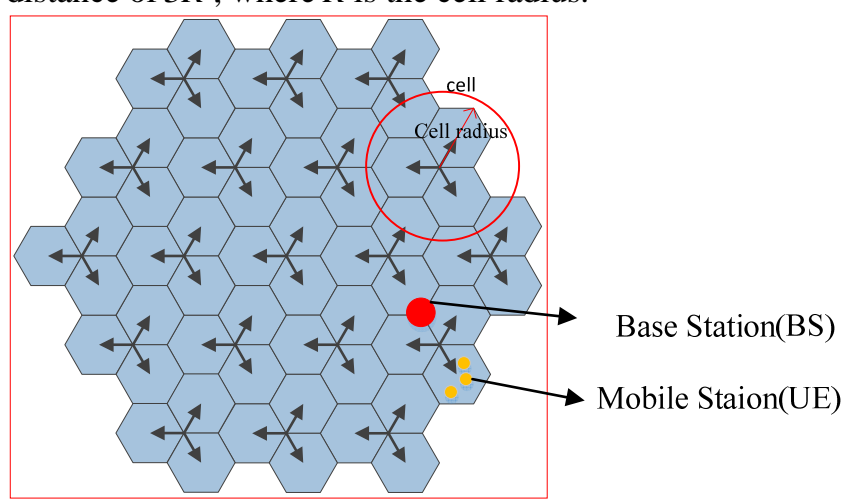

Fig. 3. Network layout

The IMT-2020 system utilizes multiple antennas when operating at high frequencies. Due to the higher path loss at high frequencies, it is important for IMT-2020 to have larger arrays compared with current LTE standards. We use array antennas with 4 columns and 8 rows in this paper. According to 3GPP modeling, the pattern of the radiation element is described as follows

$$
A_{E, H}(\phi)=-\min \left[12\left(\frac{\phi}{\phi_{3 d B}}\right)^{2}, A_{m}\right]
$$

Where $\phi$ is the azimuth angle defined between $-180^{\circ}$ and $180^{\circ} . \phi_{3 \mathrm{~dB}}$ is the horizontal $3 \mathrm{~dB}$ bandwidth. $A_{m}=30 \mathrm{~dB}$ is the front-back radio. $A_{E, H}(\phi)$ is the horizontal pattern of the radiation element.

$$
A_{E, V}(\theta)=-\min \left[12\left(\frac{\theta-90}{\theta_{3 d B}}\right)^{2}, S L A_{v}\right]
$$

Where $\theta$ is the elevation angle defined between $0^{\circ}$ and $180^{\circ} . \theta_{3 d B}$ is the vertical $3 \mathrm{~dB}$ bandwidth. SLA is the lower limit. $A_{E, V}(\theta)$ is the vertical radiation pattern of the radiation element.

$$
A_{E}(\phi, \theta)=G_{E, \text { Max }}-\min \left\{-\left[A_{E, H}(\phi)+A_{E, V}(\theta)\right], A_{m}\right\}
$$

Where $G_{E, M a x}$ is the maximum directional gain of the radiation element.
For DRS antenna pattern, we assumed a tapered circular apertures antenna with uniform distribution, described in (4) with $n=0$ [12].

$$
G_{r}\left(\theta_{a}\right)=G_{\max }\left|2^{n+1}(n+1) ! \frac{J_{n+1}\left(\theta_{a}\right)}{\left(\theta_{a}\right)^{n+1}}\right|^{2}
$$

Where a maximum antenna gain of $57.5 \mathrm{dBi}$ is deployed. Considering $\theta_{a}$ to be $10^{\circ}$, and we can get the specific value of the receiving antenna gain of DRS.

\subsection{Protection Criteria}

ITU-R Recommendation SA.1155 [13] recommends that the maximum aggregate interference power spectral density level from all sources to be exceeded for no more than $0.1 \%$ of the time be $-178 \mathrm{~dB}(\mathrm{~W} / \mathrm{kHz})$ in the 25.25 $27.5 \mathrm{GHz}$ band. This level is based on an $\mathrm{I} / \mathrm{N}=-10 \mathrm{~dB}$ and a link margin degradation of $0.4 \mathrm{~dB}$. In the reference bandwidth of $1 \mathrm{MHz}$, the maximum permissible interference level can be calculated as $-118.6 \mathrm{dBm} / \mathrm{MHz}$.

For the co-channel case the interference should not exceed $-118 \mathrm{dBm} / \mathrm{MHz}$ at the macro base station and $113 \mathrm{dBm} / \mathrm{MHz}$ at the UEs according to 3GPP TS 36.101 roughly the same as LTE terminal.

In this paper, we only consider the main beam interference, which is in the case of an IMT-base station radiating towards a DRS and coupling into the main beam of the DRS high gain antenna. The tolerable IMT-base station interference power is calculated under the assumptions of co-channel interference into a GEO DRS orbit. Assuming 3dB loss due to atmospheric absorption and $3 \mathrm{~dB}$ polarization loss, will result in interference level greater than the value specified in [14] when there is direct alignment.

\subsection{Propagation Model}

The standard model agreed upon this scenario for interference assessment is clearly denoted in the ITU-R Recommendation P.452 [15], which considers a line-ofsight (LOS) component (modeled as free space propagation). This model including the attenuation due to LOS-propagation as well as the clutter loss in different environment, is used for the frequency sharing study

$$
L_{p}=92.44+20 \lg (f)+20 \lg (d)+L_{o}
$$

Where $f$ is the operating frequency $(\mathrm{GHz})$ of the disturbed system and $d$ is the transmission distance $(\mathrm{km})$. $L_{o}$ means other losses including atmospheric absorption and polarization loss.

\section{Interference Analysis}




\subsection{Single Point Interference}

For single point interference, the interference that DRS received can be calculate as

$$
I=P_{t}+G_{t}\left(\theta_{d}\right)+G_{r}\left(\theta_{a}\right)-L_{p}
$$

Where $P_{t}$ is the transmit power of interfering system, $G_{t}\left(\theta_{d}\right)$ is the transmit antenna gain, $G_{r}\left(\theta_{a}\right)$ is the receiving antenna gain of disturbed system, $L_{p}$ is the pathloss component between the interfering system and the disturbed system. $\theta_{d}$ is the angle of departure for transmitting signals, $\theta_{a}$ is the angle of arrival for the receiving signals as illustrated in Fig. 4.

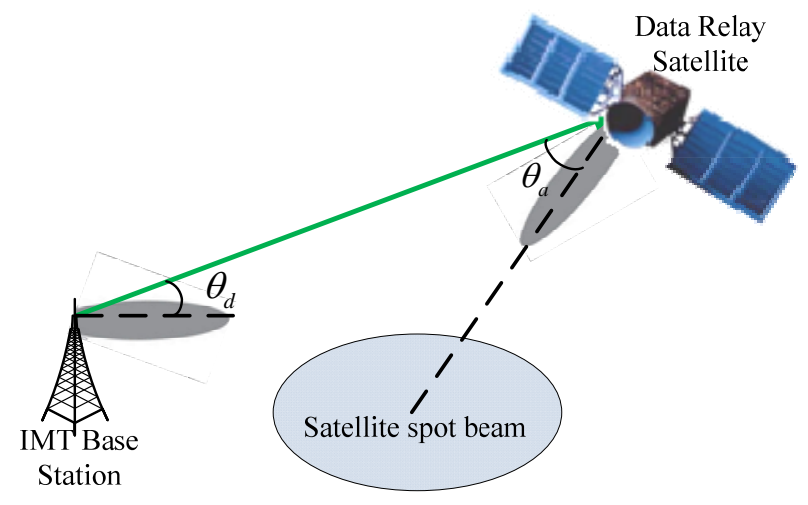

Fig. 4. Antenna radiation pattern for IMT-base station and data relay satellite

\subsection{Lumped Interference}

In the case of lumped interference, it is required to consider all the ground IMT-BSs covered by the satellite beam to calculate the lumped interference. Therefore, before calculating the lumped interference power, the location and total number of IMT-BSs should be known. Supposing in this case, each IMT BS has the same interference level to the DRS. Under the coverage of satellite, the total interference of $M$ IMT-base stations is

$$
I_{\text {lumped }}=I+10 \log (M)
$$

The number of IMT-BSs under the satellite coverage area mainly depends on three factors, the satellite coverage area $K_{\text {area }}$, city percentage $K_{\text {percentage }}$ and scatter factor per square kilometer IMT-base stations $K_{\text {scatter }}$. The total number of IMT-BSs can be obtained from the product of the three factors

$$
M=K_{\text {area }} K_{\text {percentage }} K_{\text {scatter }}
$$

An assessment of frequency sharing is based on the concept of permissible interference level at the antenna terminals of a disturbed system and only in-band operation is considered in this paper. To analyze the additional isolation, the specific calculation formula is as follows

$$
A_{\text {addiso }}=I_{\text {lumped }}-I_{\text {limit }}
$$

Where $I_{\text {limit }}$ is the permissible interference level of the disturbed system.

\subsection{The calculation of elevation angle}

For interference analysis, the elevation angle of earth station is a main parameter, when calculating the interference level. According to [16], the IMT-base station elevation angle can be calculated as

$$
\alpha=\arctan \left(\frac{\cos \left(\left|E_{E}-E_{S}\right|\right) \cos \varphi_{E}-0.151}{\sqrt{1-\cos ^{2}\left(\left|E_{E}-E_{S}\right| \cos ^{2} \varphi_{E}\right)}}\right)
$$

Where $E_{E}$ is IMT base station longitude, $E_{S}$ is DRS longitude, $\varphi_{E}$ is earth station latitude.

As the DRS user spacecraft is in low-earth orbit, when the spacecraft and UE are in different geographical position, the distance will change with the latitude and longitude of the two.

$$
d=\sqrt{r^{2}+R^{2}-2 r R \cos \left(E_{E}-E_{s}\right) \cos \varphi_{E}}
$$

Where $r$ is the radius of the earth, $R$ is the radius of satellite orbit.

\section{Simulation and Discussion}

With reference to the ITU-R Recommendation, intersatellite service system parameters are shown in Table I and IMT-2020 system parameters under consideration of sharing between the DRS in the band of $25.25-27.5 \mathrm{GHz}$ is shown in Table II.

Table 1. Inter-satellite Service System Parameters

\begin{tabular}{l|c}
\hline Parameter & Value \\
\hline Center frequency of operation [GHz] & 26.375 \\
Receiver bandwidth [MHz] & 600 \\
Maximum antenna gain/DRS [dB] & 57.5 \\
Maximum antenna gain/Spacecraft & 44.5 \\
Maximum power spectral density & -20 \\
[dBm/MHz] & \\
Feeder loss [dB] & 0 \\
\hline
\end{tabular}




\begin{tabular}{l|c}
\hline Noise temperature [K] & 100 \\
Recommend I/N level $[\mathrm{dB}]$ & -10 \\
Boltzmann constant $[\mathrm{J} / \mathrm{K}]$ & $1.38 \times 10^{-}$ \\
& \\
Noise level [dB/MHz] & -108.6 \\
Maximum permissible interference & -118.6 \\
\hline $\mathrm{dBm} / \mathrm{MHz}]$ & \\
\hline
\end{tabular}

Table 2. IMT-2020 System Parameters

\begin{tabular}{l|c}
\hline Parameter & Value \\
\hline Center frequency of operation & 26.375 \\
[GHz] & 600 \\
System bandwidth [MHz] & 200 \\
Inter-site distance [m] & 33 \\
Transmit power [dBm] & -3 \\
Feeder loss [dBm] & -3 \\
polarization isolation [dBm] & -3 \\
City base station activation rate & BS:- \\
[dBm] permissible & $118 / \mathrm{UE}:-113$ \\
Maximum & \\
interference [dBm/MHz]
\end{tabular}

For area of satellite spot beam and IMT small cell are greatly different in size, and number of IMT-BSs can do make a difference on aggregate interference assessment. We define the Effective Area Percentage (EAP) to describe the deployed IMT area in a spot beam. Assuming the longitude of DRS is $113^{\circ} \mathrm{E}$, to analyze the interference power, Fig. 5 shows the interference of IMT-BSs to DRS on different EAP and elevation angle. We observe that the interference power is always above the maximum permissible interference level making it scarcely possible to deploy IMT-2020 downlink with the return inter-orbit link of data relay satellite system.

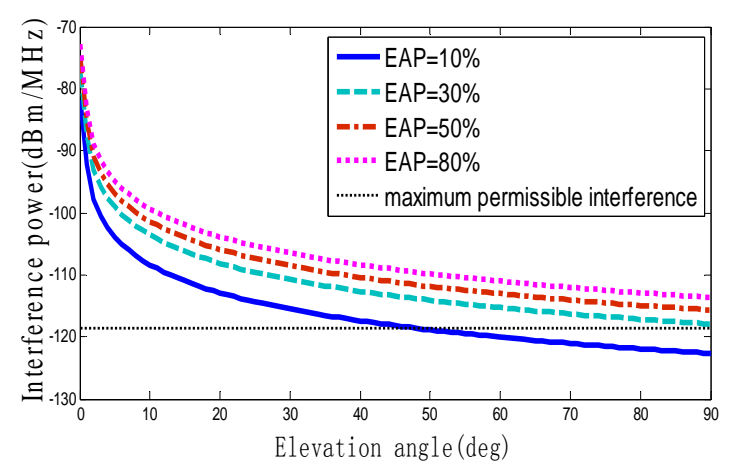

Fig. 5. Interference from IMT BSs to DRS
To evaluate the interference from spacecraft to UE, we consider the worst case scenario. Assuming the spacecraft and UE both in the same longitude, Fig. 6 shows the additional isolation required for the three cities Beijing, Harbin and Wuhan in which the latitude of three cities were $40^{\circ}, 45^{\circ}$, and $30^{\circ}$ when spacecraft at different latitude. We observe that the additional isolation is always negative which indicates the forward feeder link may coexist with IMT downlink.

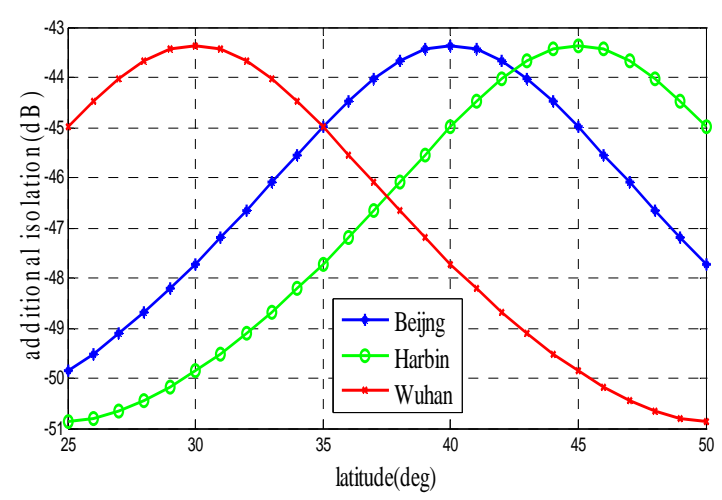

Fig. 6. Additional isolation needed for different cities

\section{Conclusion}

In this paper, we investigated frequency sharing of IMT2020 and inter-satellite service in the band between 25.25$27.5 \mathrm{GHz}$. Focusing on the interference scenario between the return inter-orbit link of data relay satellite system and downlink of IMT-2020. For the interference from IMT2020 to inter-satellite service, we analyze the interference link from IMT BSs with different elevation angles and density to DRS. For the interference from inter-satellite service to IMT-2020, we mainly evaluate the interference level from DRS user spacecraft to UE. Using the propagation model, antenna radiation patterns and parameters provided by ITU, 3GPP and other newest publications. The results from this study quantify the interference. This work can be a good reference to the research community, industry and regulators which are currently investigating spectrum requirements and technology options for 5G system. However, many challenges are still undergo. Many factors such as power control schemes and antenna deployment can do effect on the spectrum sharing between the two systems.

As future work, we intend to further study the 5G deployment and antenna technology to evaluate the interference level more accurately and provide method for the coexistence of two systems.

\section{Acknowledgements.}

This work was supported by Doctoral Scientific Fund Project of the Ministry of Education of China (20120005110001) and National Nature Science Foundation of China(91438114). 


\section{References}

[1] ITU-R Rep. M.2039, "Characteristics of terrestrial IMT2000 systems for frequency sharing/interference analyses,” Nov. 2014.

[2] Xiaoyou Yu, Yu Yang, Jinjun Ding. Satellite network design method applicable to orbitdetermination and communication for GNSS[C]. 2013 4th IEEE International Conference on Software Engineering and Service Science (ICSESS), 2013, pp:886-889.

[3] ITU-R Rec.SA.1019, "Preferred frequency bands and transmission directions for data relay satellite systems," June, 2001.

[4] ITU-R Rec.SA.1276, “Technical and operational requirements that facilitate sharing between point-to-point systems in the fixed service and the inter-satellite service in the band 25.25-27.5 GHz,” Dec.2013.

[5] T. S. Rappaport, S. Sun, R. Mayzus, H. Zhao, Y. Azar, K. Wang, G.N. Wong, J. K. Schulz, M. Samimi, F. Gutierrez, "Millimeter wave mobile communications for 5G cellular: it will work!,” IEEE Access, vol.1,pp.335-349, 2013.

[6] Joongheon Kim, Liang Xian, A. Maltsev, R. Arefi and A. S. Sadri, "Study of coexistence between 5G small-cell systems and systems of the fixed service at $39 \mathrm{GHz}$ band," 2015 IEEE MTT-S International Microwave Symposium, Phoenix, AZ, 2015, pp. 1-3.

[7] F. Guidolin and M. Nekovee, "Investigating Spectrum Sharing between 5G Millimeter Wave Networks and Fixed Satellite Systems," 2015 IEEE Globecom Workshops (GC Wkshps), San Diego, CA, 2015, pp. 1-7.

[8] ITU-R Rec. F.1249, "Technical and operational requirements that facilitate sharing between point-to-point systems in the fixed service and the inter-satellite service in the band 25.25-27.5 GHz,” Sep.2015.

[9] ITU-R Rec SA.1018, "Hypothetical reference system for systems comprising data relay satellites in the geostationary orbit and user spacecraft in low Earth-orbits," Mar.1994.

[10] Tercero M, Sharma S, Coldrey M, et al. Coexistence between 5G and Fixed Services[C]//Vehicular Technology Conference (VTC Spring), 2016 IEEE 83rd. IEEE, 2016: 1-5.

[11] Rusek F, Persson D, Lau B K, et al. Scaling up MIMO: Opportunities and challenges with very large arrays[J]. IEEE Signal Processing Magazine, 2013, 30(1): 40-60.

[12] ITU-R Rec F.1336, "Reference radiation patterns of omnidirectional, sectoral and other antennas for the fixed and mobile services for use in sharing studies in the frequency range from $400 \mathrm{MHz}$ to about $70 \mathrm{GHz}$," Feb. 2014.

[13] ITU-R Rec SA.1155, "Protection criteria related to the operation of data relay satellite systems," Dec.2013.

[14] W. L. Stutzman and G. A. Thiele, Antenna Theory and Design, 3nd ed., New York: John Wiley and Sons, 2012, p. 389.

[15] ITU-R Rec P.452, "Prediction procedure for the evaluation of interference between stations on the surface of the Earth at frequencies above about $0.1 \mathrm{GHz}$,” July.2015.

[16] Electromagnetic Spectrum Engineering. Jingchun Li, Biao Huang, Jia Huang. Posts\&Telecom Press. June 2008. 